\title{
Autologous platelet-rich plasma promotes proliferation and chondrogenic differentiation of adipose-derived stem cells
}

\author{
JI SHEN ${ }^{1,2}$, QINGFENG GAO ${ }^{1,2}$, YAO ZHANG ${ }^{1,2}$ and YAOHUA HE YH, $^{1,2}$ \\ ${ }^{1}$ Department of Orthopaedics, Shanghai Jiao Tong University Affiliated Sixth People's Hospital, Shanghai 200233; \\ ${ }^{2}$ Department of Orthopaedics, Shanghai Sixth People's Hospital, Soochow University, Soochow, Jiangsu 215000, P.R. China
}

Received December 15, 2013; Accepted August 29, 2014

DOI: $10.3892 / \mathrm{mmr} .2014 .2875$

\begin{abstract}
Cartilage regeneration is a promising potential therapy for articular cartilage defects and adult stem cells serve a key role in regenerative medicine. Adipose-derived stem cells (ADSCs) have been identified as an alternative source of adult stem cells in recent years and can be differentiated into numerous types of cell, including chondrocytes, adipocytes and osteoblasts. However, their clinical use is restricted by the proliferation of cells, and their tendency to dedifferentiate. Platelet-rich plasma (PRP) has recently emerged as a potential bioactive material to promote cell proliferation and differentiation, based on the release of growth factors. In the current study, the effect of autologous PRP on the proliferation and chondrogenic differentiation of ADSCs was examined. The results indicated that PRP promotes ADSC proliferation and suggested that PRP leads to chondrogenic differentiation of ADSCs in vitro. When co-cultured with chondrocytes, the ADSCs on three-dimensional PRP scaffolds were able to form neocartilage, with positive staining of safranine $\mathrm{O}$, which indicated the production of glycosaminoglycan, and type II collagen.
\end{abstract}

\section{Introduction}

There are thousands of patients suffering from articular cartilage defects worldwide, creating a great economic burden on society. The repair of articular cartilage defects remains a challenge for orthopedists, although a series of methods have been developed to attempt to solve these problems, including chondrocyte transplantation, cartilage transplantation and artificial joint replacement (1). Cartilage tissue engineering provides a novel method for the repair of articular cartilage defects, an alternative to the traditional 'Traumatic

Correspondence to: Dr Yaohua He, Department of Orthopaedics, Shanghai Jiao Tong University Affiliated Sixth People's Hospital, 600 Yi Shan Road, Shanghai 200233, P.R. China

E-mail: heyaohua@vip.163.com

Key words: platelet-rich plasma, adipose-derived stem cells, cartilage regeneration
Restorative Treatment' mode, which results in the treatment of lesions at the cost of creating another lesions; for example, the reconstruction of the ear in microtia patients where the costal cartilage was excised and used for reconstruction of the ear (2). Adipose-derived stem cells (ADSCs) are a notable alternative source of seed cells for cartilage tissue engineering. They can be easily collected by liposuction and have the potential to differentiate into various types of mesenchymal cell, including osteoblast, chondrocyte and muscle cells (3-5). Yao et al (6) reported ectopic bone formation in adipose-derived stromal cell-seeded osteoinductive calcium phosphate scaffolds. Additionally, Yoon et al (7) demonstrated enhanced cartilage formation via three-dimensional cell engineering of human ADSCs. Besides the seeding cells, the biomaterial scaffold is also essential to the success of cartilage tissue engineering applications. Platelet-rich plasma (PRP) is blood plasma with increased levels of platelets. As a concentrated source of autologous platelets, PRP contains a high level of several growth factors and other cytokines that have been widely used in orthopedic surgery and regenerative medicine (8-10). The significant advantages of PRP over other biomaterials are its autologous source and high concentration of growth factors, including transforming growth factor (TGF) $\beta$, insulin-like growth factor (IGF) and vascular endothelial growth factor $(8,11)$. Previously, PRP was utilized in bone graft augmentation in oral and maxillofacial surgery, and the results indicated significantly improved bone density and fusion rates in the mandible (12). PRP has potential uses in tissue healing and regeneration. Coviello et al (13) evaluated PRP wound healing benefits in multiple myeloma (MM) patients that developed osteonecrosis of the jaw (ONJ) following surgical tooth extraction, and the results indicated that PRP improves wound healing in MM bisphosphonate-associated ONJ. Also, Driver et al (14) reported autologous PRP gel treatment of nonhealing diabetic foot ulcers, and observed that PRP contributed to the healing process of these chronic wounds. In vitro studies have confirmed that PRP enhances the proliferation of a variety of human cell types. In addition, a number of studies indicated that PRP increases cell growth and the synthesis of extracellular matrix. Lucarelli et al (15) observed that 10\% PRP promotes bone marrow-derived stem cell proliferation, and Akeda et al (16) also reported that PRP stimulates porcine chondrocyte proliferation and matrix biosynthesis. 
In the current study, the effect of PRP on the proliferation and chondrogenic differentiation of ADSCs was investigated in order to evaluate the potential application of ADSCs and PRP in the repair of articular cartilage defects. The concentration of different growth factors and the proliferation of ADSCs were analyzed in the present study, and the expression levels of selected cartilage-specific genes were evaluated in order to specifically explore the chondrogenic differentiation of ADSCs in the presence or absence of PRP. The present study further investigated the in vivo neocartilage formation of ADSCs in PRP gel when co-cultured with chondrocytes.

\section{Materials and methods}

Tissue samples. The adipose tissue samples were obtained from the lipoaspirates of patients who had undergone liposuction (mean age, 27 years; range, 20-35 years). Chondrocytes were isolated from articular cartilage slices of patients (mean age, 58 years; range, 49-62 years) undergoing total knee arthroplasty at Shanghai 6th People's Hospital (Shanghai, China) from August 2012 to August 2013.

Cell harvest and culture. ADSCs from lipoaspirates and chondrocytes from articular cartilage were isolated and expanded as previously described $(5,17)$. ADSCs and chondrocytes at passage 3 were used in the present study.

PRP preparation. Human PRP was harvested from the peripheral blood using a continuous two-step sedimentation process as previously described (18). Briefly, $10 \mathrm{ml}$ peripheral blood was harvested from cubital fossa venipuncture into vacuum tubes and centrifuged at $200 \mathrm{x}$ g for $10 \mathrm{~min}$. Next, the plasma was separated into PRP by further centrifugation at $200 \mathrm{x} \mathrm{g}$ for $10 \mathrm{~min}$. To activate the PRP, $10 \%$ thrombin solution (v/v, $1,000 \mathrm{U} / \mathrm{ml}$ in $100 \mathrm{mM} \mathrm{CaCl}_{2}$ ) was added to the PRP to yield PRP gel. Soluble PRP releasates from the clotted preparations were isolated by centrifugation (1,000 $\mathrm{x} \mathrm{g}$ for $5 \mathrm{~min})$.

Detection of growth factors. It is established that growth factors are important in the proliferation and chondrogenic differentiation of ADSCs, thus an enzyme-linked immunosorbent assay (ELISA) was utilized to quantitatively analyze the concentrations of the growth factors TGF- $\beta$, platelet-derived growth factor BB (PDGF-BB), IGF and epidermal growth factor (EGF) in the whole blood and in the activated PRP.

Measurement of cell proliferation. The ADSCs were cultured under four different conditions as follows: $10 \%$ fetal bovine serum (FBS), 5\% PRP (PRP releasate in serum-free medium), $10 \%$ PRP and $20 \%$ PRP. The proliferation of ADSCs was measured by a cell counting kit assay (CCK8; Dojindo, Rockville, MD, USA) following culture in different concentrations of PRP on days 1, 3, 5 and 7 in vitro, as described in a previous study (19).

Reverse transcription-quantitative polymerase chain reaction (RT-qPCR) analysis. Cells from the four groups were harvested at days 1, 3 and 7 then dissolved in TRIzol (Sigma-Aldrich, St. Louis, MO, USA) to extract the total RNA according to previously described methods (20). In brief, Total RNA was extracted
Table I. Primers used for quantitative polymerase chain reaction.

\begin{tabular}{llc}
\hline Gene & \multicolumn{1}{c}{ Primer } & Product (bp) \\
\hline $\begin{array}{l}\text { Type II } \\
\text { collagen }\end{array}$ & F-CTGGACTAGTGGGTCCCAGG & 111 \\
Sox-9 & F-GGCTCTCCTTGCTCACCCTTG & \\
& R-GTGCGGCTTATTCTTGCTCG & 195 \\
Aggrecan F-GGTGTAGGCACCTCCTTTCC & 106 \\
& R-GAAAGGGTGAGGGGTGTCAG & \\
\multirow{3}{*}{-actin } & F-CTTCCAGCCTTCCTTCCTGG & 110 \\
& R-CTGTGTTGGCGTACAGGTCT & \\
&
\end{tabular}

F, forward; R, reverse.

using Trizol according to the manufacturer's instructions. $1 \mu \mathrm{g}$ RNA was reverse-transcribed into cDNA using Superscript II reverse transcriptase (Invitrogen Life Technologies, Carlsbad, CA, USA) according to the manufacturer's instructions. cDNA amplification was performed in a thermocycler (Biometra T3000; Biometra, Göttingen, Germany) using Taq polymerase supplied with $\mathrm{KCl}$ buffer and $1.5 \mathrm{mM} \mathrm{MgCl} 2$ (Invitrogen Life Technologies) at $94^{\circ} \mathrm{C}$ for $1 \mathrm{~min}, 58^{\circ} \mathrm{C}$ for $30 \mathrm{sec}$ and $72^{\circ} \mathrm{C}$ for $1 \mathrm{~min}$. PCR products were resolved on $1.5 \%$ agarose gel (Invitrogen Life Technologies) run in $1 \mathrm{X}$ Tris borate-EDTA buffer. The expression levels of the genes were quantified in duplicates, using SYBR Green Master Mix (Applied Biosystems, Foster City, CA, USA). PCR reactions were run on an ABI 7900HT RT-PCR system (Applied Biosystems) and the SDS software, version 2.1 (Applied Biosystems) was used to analyze the results. Gene expression was analyzed via comparative CT Method $\left({ }^{\triangle \Delta C T}\right)$ and were normalized to 18 s rRNA. qPCR was used to detect expression levels of cartilage-specific genes (type II collagen, sox-9 and aggrecan) in order to evaluate the level of chondrogenic differentiation. The primer sequences are presented in Table I.

Construction of ADSC/PRP composites. The ADSCs at passage 3 were used for constructing ADSC/PRP composites. Briefly, the cells were counted and resuspended in PRP, at a cell density of $5.0 \times 10^{6} / \mathrm{ml}$. Next, $0.15 \mathrm{ml}$ of $10 \%$ thrombin solution (v/v, 1,000 U/ml in $100 \mathrm{mM} \mathrm{CaCl}_{2}$; Sigma-Aldrich) was added to the cell/PRP suspension to activate the PRP and form ADSC/PRP composites. The ADSC/PRP composites were divided into two groups: The experimental group (ADSC/PRP composite co-culture with chondrocytes); and the control group (ADSC/PRP composites alone). In the experimental group, the ADSC/PRP composites were cultured in high-glucose HyClone Dulbecco's modified Eagle's medium containing 10\% FBS (GE Healthcare Bio-Sciences, Piscataway, PA, USA) in a $0.4-\mu \mathrm{m}$ Transwell ${ }^{\circledR}$ chamber (Corning Life Sciences, Corning, NY, USA) with adherent chondrocytes cultured in the plate below the membrane, while ADSC/PRP composites in the control group were cultured in the medium alone. After 21 days, the composites were implanted subcutaneously into BALB-c nude mice (four weeks old; weight, $20 \mathrm{~g}$ ). Animals were sacrificed by cervival dislocation and the samples were harvested at 

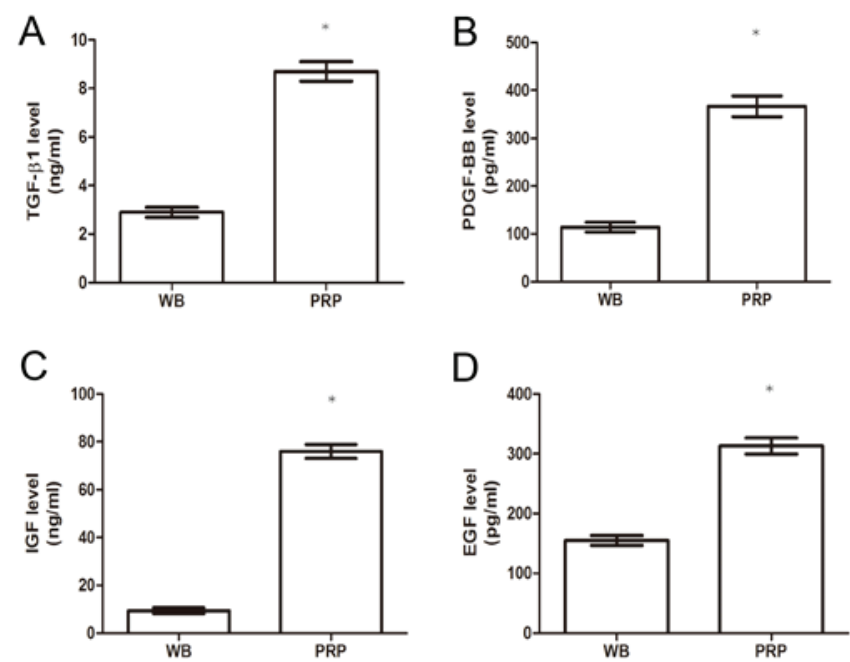

Figure 1. Detection and quantification of growth factor expression levels. The concentrations of (A) TGF- $\beta 1$, (B) PDGF-BB, (C) IGF and (D) EGF were significantly increased in PRP ("P<0.05 vs. WB). WB, whole blood; PRP, platelet-rich plasma; TGF- $\beta 1$, transforming growth factor- $\beta 1$; PDGF-BB, platelet-derived growth factor BB; IGF, insulin-like growth factor; EGF; epidermal growth factor.

8 weeks post-implantation. Mice were purchased from the Shanghai Animal Center (Shanghai, China) and all animal experimental procedures in the present study were approved by the Ethics Committee of Shanghai Jiao Tong University School of Medicine (Shanghai, China). Mice were housed at $21^{\circ} \mathrm{C}$ in regular light (06:30-19:30 h)-dark (19:30-06:30 h) cycles with food and water ad libitum at the Animal Care and Veterinary Services Facility, according to the guidelines of the International Council for Laboratory Animal Science. They were acclimated for 2 weeks prior to the experiment.

Histological examination. Specimens were fixed in neutral-buffered formalin overnight at $4^{\circ} \mathrm{C}$, embedded in paraffin and sectioned (5- $\mu \mathrm{m}$ thickness). The cross-sections were stained with hematoxylin/eosin and safranin-O dyes, which indicate production of glycosaminoglycan, then observed under an inverted phase contrast microscope (TS100; Nikon, Tokyo, Japan).

Immunohistochemical analysis. Collagen II immunohistochemical staining was performed to evaluate the chondrogenic differentiation of ADSC/PRP composites. The sections were immersed in phosphate-buffered saline (PBS) containing $1 \%$ goat serum at room temperature for $2 \mathrm{~h}$ to block non-specific reactions. Subsequently, the sections were incubated in PBS containing $1 \%$ bovine serum albumin (BSA) and collagen type II monoclonal clonal mouse anti-rabbit antibody (DAB; Santa Cruz Biotechnology, Inc., Dallas, TX, USA) at $4^{\circ} \mathrm{C}$ overnight. Following washing with PBS three times, the samples were incubated in PBS containing 3\% BSA. Finally, the samples were incubated in PBS containing 1\% BSA and horseradish peroxidase (HRP)-conjugated anti-mouse immunoglobulin $\mathrm{G}$ antibody (1:200; Santa Cruz Biotechnology, Inc.) at $25^{\circ} \mathrm{C}$ for 4 $\mathrm{h}$, followed by color development with diaminobenzidine tetrahydrochloride (Santa Cruz Bitoechnology, Inc.) (21).

Biochemical and biomechanical assay. After 8 weeks of in vivo culture, the compressive modulus, glycosaminoglycan (GAG) content and total collagen content were determined according to previously described methods (22). In brief, a biomechanical analyzer (Instron, Canton, MA, USA) was used for biomechanical test, in which a constant compressive strain rate of $1 \mathrm{~mm} / \mathrm{min}$ was applied until a maximal force of $100 \mathrm{~N}$ was achieved and thus a force-displacement curve was obtained. The compressive modulus of tested tissue was calculated based on the force-displacement curve.

Statistical analysis. Data are presented as the mean \pm standard deviation. The data were analyzed by one-way analysis of variance using SPSS 17.0 software (International Business Machines, Armonk, NY, USA). $\mathrm{P}<0.05$ was considered to indicated a statistically significant difference.

\section{Results}

Quantification of growth factors. As presented in Fig. 1, the levels of the following growth factors exhibited a significant difference between the two groups: TGF- $\beta 1$ $(8.70 \pm 1.10 \mathrm{ng} / \mathrm{ml}$ in PRP vs. $2.90 \pm 0.51 \mathrm{ng} / \mathrm{ml}$ in whole blood); PDGF-BB $(367.00 \pm 53.21 \mathrm{pg} / \mathrm{ml}$ vs. $114.43 \pm 25.35 \mathrm{pg} / \mathrm{ml})$; IGF $(76.00 \pm 8.22 \mathrm{ng} / \mathrm{ml}$ vs. $9.40 \pm 1.60 \mathrm{ng} / \mathrm{ml})$; and EGF $(313 \pm 33.65 \mathrm{pg} / \mathrm{ml}$ vs. $155 \pm 23.98 \mathrm{pg} / \mathrm{ml})$, indicating that activated PRP contained higher levels of the growth factors than whole blood.

ADSC proliferation in different concentrations of PRP. Fig. 2 indicates the results of the cell proliferation assay. A significant increase in the number of ADSCs over the 7-day in vitro culture period was observed. In addition, there was a general increase in cell number with increasing concentrations of PRP, and 20\% PRP had the strongest effect on ADSC proliferation.

Expression of cartilage-specific genes. Expression levels of chondrogenic differentiation-related genes were significantly increased following PRP incubation. Collagen II, sox-9 and aggrecan were upregulated in a dose- and time-dependent manner (Fig. 3). 
A

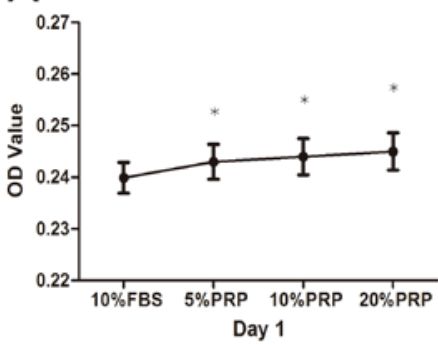

C

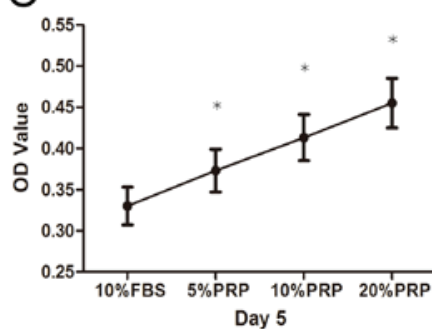

B

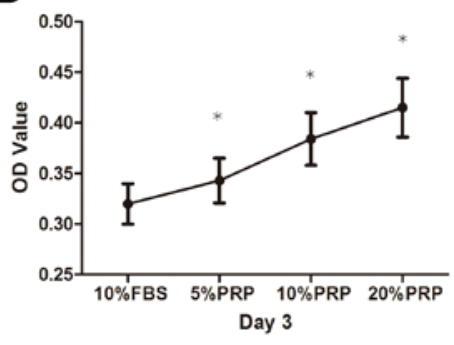

D

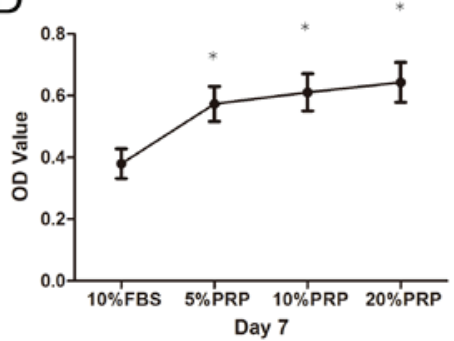

Figure 2. In vitro proliferation of ADSCs. ADSCs were cultured in different conditions over different time periods (A) day 1, (B) day 3, (C) day 5 and (D) day 7 . With increasing PRP concentration, the level of cell proliferation increased ( $\mathrm{P}<0.05)$. OD, optical density; ADSC, adipose-derived stem cell; FBS, fetal bovine serum; PRP, platelet-rich plasma.
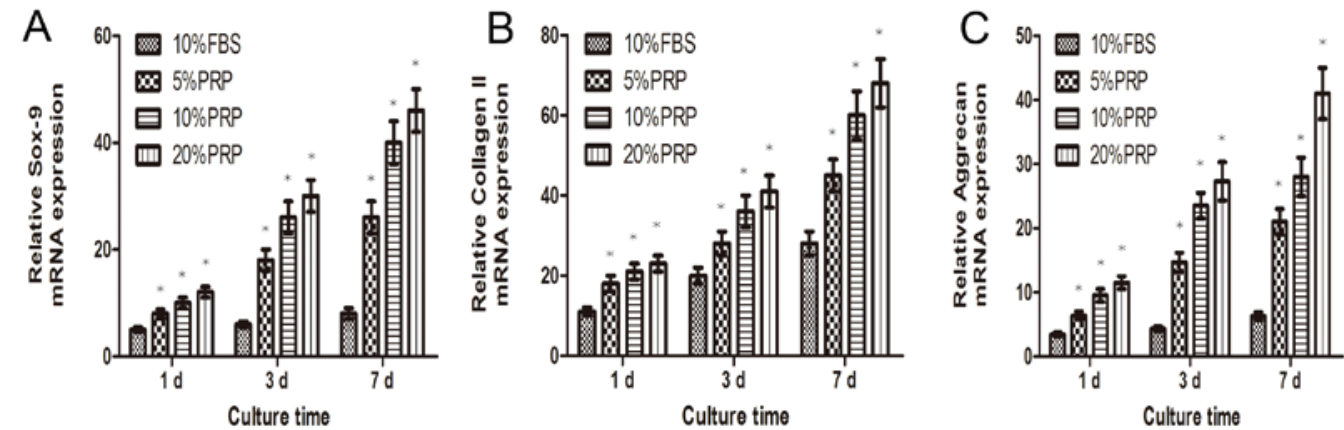

Figure 3. Reverse transcription-quantitative polymerase chain reaction demonstrated that PRP treatment led to an increase in the expression of cartilage-specific genes (collagen II, sox-9 and aggrecan) of ADSCs in a dose and time-dependent manner ( $\mathrm{P}<0.05$ ). ADSC, adipose-derived stem cell; FBS, fetal bovine serum; PRP, platelet-rich plasma.

Invivo resultof ADSC/PRP composites. Eight weeks subsequent to subcutaneous implantation, the ADSC/PRP composites in the experimental group presented an ivory-whitish cartilage-like appearance, while the composites in the control group had shrunk and become fibrous.

The results of histological and immunohistochemical examinations showed that the composites in the experimental group formed cartilage-like tissue with obvious lacuna-like structures, and positive staining for safranin $\mathrm{O}$, indicating the production of glycosaminoglycan, and type II collagen (Fig. 4). The results of the biochemical and biomechanical assays indicated that the GAG and total collagen content, in addition to the compressive modulus, were significantly increased in the PRP-treated groups when compared with the control group $(\mathrm{P}<0.05$; Fig. 5).

\section{Discussion}

Cartilage tissue engineering provides a potential approach for the repair of articular cartilage defects (23). Cartilage tissue engineering requires three key elements: A seeding cell source, a three-dimensional biomaterial scaffold and a chondrogenic microenvironment (24). Adult stem cells may be ideal donor cells in cartilage regeneration, and previous studies on the bone marrow-derived stem cells (BMSCs) have been conducted (25). Liu et al (24) constructed mature engineered cartilage in vitro and in vivo using BMSCs (26). However, the limited supply of BMSCs restricts the clinical application of BMSC-based engineered cartilage tissue. By contrast, adipose tissue, with its abundant sources and easy methods of acquisition, in addition to possessing multilineage differentiation potential and high proliferation potential in vitro, is becoming an attractive seeding cell source for tissue engineering (27). Animal and clinical studies have previously demonstrated that ADSCs were able to repair damaged skeletal tissue or bone defects. Declercq et al (28) constructed bone grafts engineered from human ADSCs in dynamic three-dimensional environments, and Guasti et al (29) reported chondrogenic differentiation of ADSCs within nanocaged POSS-PCU scaffolds. In the 


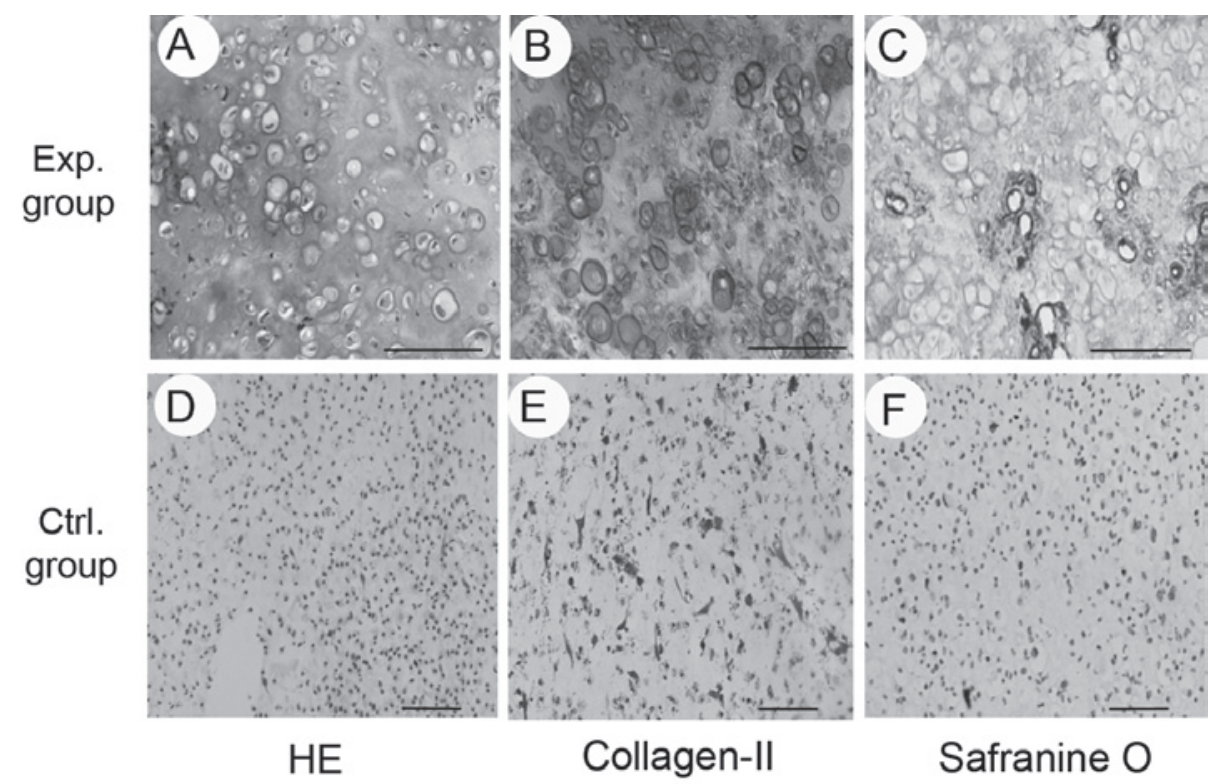

Figure 4. Histological and immunohistochemical evaluation. (A) Lacuna-like structures, (B) positive safranin O staining and (C) type II collagen immunostaining were observed in the ADSC/PRP composites co-cultured with chondrocytes, indicating that the constructs had formed cartilage-like tissue, while ADSC/PRP composites alone formed fibrous tissue(D-F). Scale bar=100 $\mu \mathrm{m}$. ADSC, adipose-derived stem cell; PRP, platelet-rich plasma; HE, hematoxylin \& eosin.
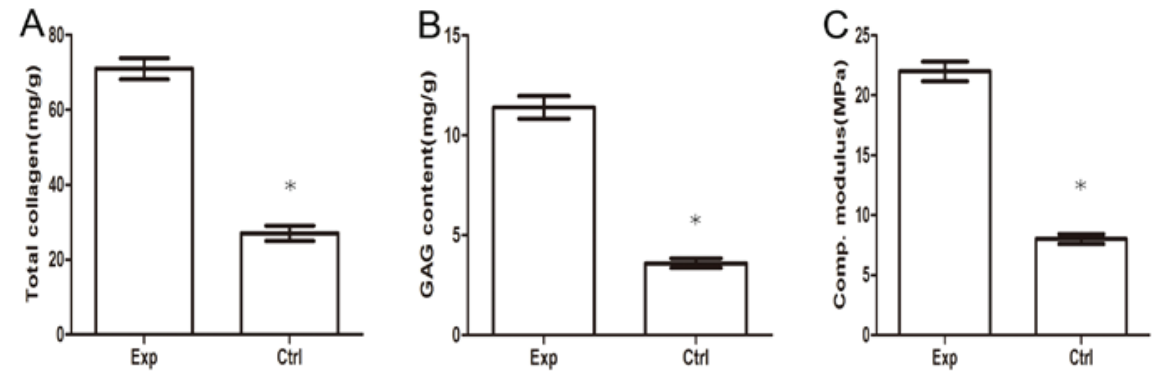

Figure 5. Biochemical and biomechanical assay. (A) Collagen levels; (B) GAG levels; and (C) compressive modulus. Values were increased in the experimental group compared with the controls ( $\mathrm{P}<0.05)$. In the experimental group, the ADSC/PRP composites were cultured in high-glucose HyClone Dulbecco's modified Eagle's medium (containing 10\% FBS) in a $0.4 \mu \mathrm{m}$ Transwell@ chamber, with adherent chondrocytes cultured in the plate below the membrane. After 21 days in vitro culture, the composites were implanted subcutaneously into BALB-c nude mice. Animals were sacrificed by cervival dislocation and the samples were harvested 8 weeks after implantation. GAG, glycosaminoglycan.

current study, the chondrogenic differentiation of ADSCs was also demonstrated.

The other key aspect of cartilage tissue engineering is the biomaterial scaffold. The quality of the scaffold contributes greatly to the pattern of cell growth, proliferation and differentiation on the scaffold and the success of tissue regeneration. Previously, PRP, as autologous blood plasma containing high levels of growth factors, has been widely used in regenerative medicine, promoting the proliferation and differentiation of a variety of cell types, including chondrocytes and mesenchymal stem cells (MSCs) $(15,16)$. In the present study, the levels of the different growth factors were measured in whole blood and PRP, and the results indicated that the concentrations of growth factors in PRP were significantly higher than the levels in whole blood. PRP, as a concentrated source of autologous platelets, contains several different growth factors and the release of growth factors occurs following the activation of
PRP. Conventionally, the addition of calcium and/or thrombin to PRP is used to promote the release of the $\alpha$ granules from platelets, and this process creates a PRP gel that contains high concentrations of growth factors, such as TGF- $\beta 1$, IGF, EGF and PDGF-BB.

The release of growth factors contributes to cell proliferation and differentiation. The growth curve of ADSCs in different levels of PRP indicates that PRP promotes ADSC proliferation, and ADSCs in PRP exhibited increased growth rates than those in 10\% FBS. Kakudo et al (30) demonstrated that ADSCs more rapidly proliferate in 5\% activated PRP than in $20 \%$ activated PRP. However, the present results indicated that ADSC proliferation increased in a PRP concentration-dependent manner.

Additionally, PRP stimulates ADSC proliferation and chondrogenic differentiation of ADSCs in vitro. The current in vitro findings suggested that cartilage-specific genes, such 
as collagen II, sox-9 and aggrecan were also significantly higher in the PRP-treated cells compared with the controls. However, the results also indicated that the ADSC/PRP composites alone were not able to form neo-cartilage 8 weeks following subcutaneous implantation. This indicates that the expression of cartilage-specific genes was reduced and suggests that the expression of growth factors and other cytokines was decreased, and were therefore unable to promote the chondrogenic differentiation of ADSCs. Notably, ADSCs in the PRP gel formed neo-cartilage in vivo when co-cultured with chondrocytes, while ADSCs combined with PRP gel alone formed fibrous tissue in vivo. A previous study reported that chondrocytes promoted the chondrogenic differentiation of MSCs. Xue et al (21) demonstrated that chondrocytes promoted the stable subcutaneous chondrogenesis of bone marrow-derived stromal cells. This suggests that PRP may promote the chondrogenic differentiation of ADSC in a chondrogenic microenvironment and particularly in an articular microenvironment, indicating that ADSCs combined with PRP gel would be an ideal therapy for the repair of articular cartilage defects.

In conclusion, the current study identified that inactivated PRP significantly enhances human ADSC proliferation in vitro. In addition, it was demonstrated that inactivated PRP promoted the expression of cartilage-specific genes, including collagen II, sox-9 and aggrecan in vitro. ADSCs combined with PRP gel formed mature engineered cartilage tissue in vivo following co-culture with chondrocytes, providing a potential novel method for the treatment of cartilage defects.

\section{Acknowledgements}

This study was supported by the National Natural Science Foundation of China (grant nos. 81071452 and 81271961).

\section{References}

1. Mera H, Itokazu M and Wakitani S: Cartilage repair and regenerative medicine; past, present, and future. Clin Calcium 23: 1715-1722, 2013 (In Chinese).

2. Kristiansen M, Öberg M, Wikström SO: Patients' satisfaction after ear reconstruction with autologous rib cartilage. J Plast Surg Hand Surg 47: 113-117, 2013.

3. Liu G, Cheng Y, Guo S, et al: Transplantation of adipose-derived stem cells for peripheral nerve repair. Int J Mol Med 28: 565-572, 2011.

4. Cho JW, Kang MC and Lee KS: TGF- $\beta 1$-treated ADSCs-CM promotes expression of type I collagen and MMP-1, migration of human skin fibroblasts, and wound healing in vitro and in vivo. Int J Mol Med 26: 901-906, 2010.

5. Lv XJ, Zhou GD, Liu Y, et al: In vitro proliferation and differentiation of adipose-derived stem cells isolated using anti-CD105 magnetic beads. Int J Mol Med 30: 826-834, 2012.

6. Yao J, Li X, Bao C, et al: Ectopic bone formation in adipose-derived stromal cell-seeded osteoinductive calcium phosphate scaffolds. J Biomater Appl 24: 607-624, 2010.

7. Yoon HH, Bhang SH, Shin JY, Shin J and Kim BS: Enhanced cartilage formation via three-dimensional cell engineering of human adipose-derived stem cells. Tissue Eng Part A 18: 1949-1956, 2012.

8. Fallouh L, Nakagawa K, Sasho T, et al: Effects of autologous platelet-rich plasma on cell viability and collagen synthesis in injured human anterior cruciate ligament. J Bone Joint Surg Am 92: 2909-2916, 2010.

9. Kang YH, Jeon SH, Park JY, et al: Platelet-rich fibrin is a Bioscaffold and reservoir of growth factors for tissue regeneration. Tissue Eng Part A 17: 349-359, 2011.
10. Vadalà G, Di Martino A, Tirindelli MC, Denaro L and Denaro V: Use of autologous bone marrow cells concentrate enriched with platelet-rich fibrin on corticocancellous bone allograft for posterolateral multilevel cervical fusion. J Tissue Eng Regen Med 2: 515-520, 2008.

11. Cho JW, Kim SA and Lee KS: Platelet-rich plasma induces increased expression of G1 cell cycle regulators, type I collagen, and matrix metalloproteinase-1 in human skin fibroblasts. Int J Mol Med 29: 32-36, 2012.

12. Marx RE, Kline SN, Johnson RP, et al: The use of freeze-dried allogeneic bone in oral and maxillofacial surgery. J Oral Surg 39: 264-274, 1981.

13. Coviello V, Peluso F, Dehkhargani SZ, et al: Platelet-rich plasma improves wound healing in multiple myeloma bisphosphonate-associated osteonecrosis of the jaw patients. J Biol Regul Homeost Agents 26: 151-155, 2012.

14. Driver VR, Hanft J, Fylling CP and Beriou JM; Autologel Diabetic Foot Ulcer Study Group: A prospective, randomized, controlled trial of autologous platelet-rich plasma gel for the treatment of diabetic foot ulcers. Ostomy Wound Manage 52: 68-74, 2006.

15. Lucarelli E, Beccheroni A, Donati D, et al: Platelet-derived growth factors enhance proliferation of human stromal stem cells. Biomaterials 24: 3095-3100, 2003.

16. Akeda K, An HS, Okuma M, et al: Platelet-rich plasma stimulates porcine articular chondrocyte proliferation and matrix biosynthesis. Osteoarthritis Cartilage 14: 1272-1280, 2006.

17. Moon MH, Jeong JK, Lee YJ, Seol JW and Park SY: Sphingosine-1-phosphate inhibits interleukin-1 $\beta$-induced inflammation in human articular chondrocytes. Int J Mol Med 30: 1451-1458, 2012.

18. Kim YH, Furuya H and Tabata Y: Enhancement of bone regeneration by dual release of a macrophage recruitment agent and platelet-rich plasma from gelatin hydrogels. Biomaterials 35: 214-224, 2014

19. Van Pham P, Bui KH, Ngo DQ, et al: Activated platelet-rich plasma improves adipose-derived stem cell transplantation efficiency in injured articular cartilage. Stem Cell Res Ther 4: 91, 2013.

20. Xue K, Qi L, Zhou G and Liu K: A two-step method of constructing mature cartilage using bone marrow-derived mesenchymal stem cells. Cells Tissues Organs 197: 484-495, 2013.

21. Xue K, Zhu Y, Zhang Y, Chiang C, Zhou G and Liu K: Xenogeneic chondrocytes promote stable subcutaneous chondrogenesis of bone marrow-derived stromal cells. Int J Mol Med 29: 146-152, 2012.

22. Wu J, Xue K, Li H, Sun J and Liu K: Improvement of PHBV scaffolds with bioglass for cartilage tissue engineering. PloS One 8: e71563, 2013.

23. Cima LG, Vacanti JP, Vacanti C, Ingber D, Mooney D and Langer R: Tissue engineering by cell transplantation using degradable polymer substrates. J Biomech Eng 113: 143-151, 1991.

24. Vinatier C, Bouffi C, Merceron C, et al: Cartilage tissue engineering: towards a biomaterial-assisted mesenchymal stem cell therapy. Curr Stem Cell Res Ther 4: 318-329, 2009.

25. Dorotka R, Windberger U, Macfelda K, Bindreiter U, Toma C and Nehrer S: Repair of articular cartilage defects treated by microfracture and a three-dimensional collagen matrix. Biomaterials 26: 3617-3629, 2005.

26. Liu K, Zhou GD, Liu W, et al: The dependence of in vivo stable ectopic chondrogenesis by human mesenchymal stem cells on chondrogenic differentiation in vitro. Biomaterials 29: 2183-2192, 2008.

27. Qing W, Guang-Xing C, Lin G and Liu Y: The osteogenic study of tissue engineering bone with BMP2 and BMP7 gene-modified rat adipose-derived stem cell. J Biomed Biotechnol 2012: 410879, 2012.

28. Declercq HA, De Caluwé T, Krysko O, Bachert $\mathrm{C}$ and Cornelissen $\mathrm{MJ}$ : Bone grafts engineered from human adipose-derived stem cells in dynamic 3D-environments. Biomaterials 34: 1004-1017, 2013.

29. Guasti L, Vagaska B, Bulstrode NW, Seifalian AM and Ferretti P: Chondrogenic differentiation of adipose tissue-derived stem cells within nanocaged POSS-PCU scaffolds: a new tool for nanomedicine. Nanomedicine 10: 279-289, 2013.

30. Kakudo N, Minakata T, Mitsui T, Kushida S, Notodihardjo FZ and Kusumoto K: Proliferation-promoting effect of platelet-rich plasma on human adipose-derived stem cells and human dermal fibroblasts. Plast Reconstr Surg 4:1352-1360, 2008. 